\title{
POPULATION GENETICS OF SOCIAL HYMENOPTERA WITH WORKER PRODUCED MALES
}

\author{
ROBIN E. OWEN \\ Ramsay Wright Zoological Laboratories, University of Toronto, \\ Toronto, Ontario M5S 1A1, Canada
}

Received 28.viii.79

\section{SUMMARY}

\begin{abstract}
In many social Hymenoptera, particularly the bees and wasps, a proportion, $\psi$, of the males are produced by the workers. Using deterministic theory for a single locus it is shown that having worker produced males affects the speed of approach to Hardy-Weinberg equilibrium, the equilibrium gene frequency under mutation with counter-selection, the speed of elimination of a deleterious allele and the rate of advance of an advantageous gene. Equilibrium gene frequencies of a balanced polymorphism are shifted by a positive value of $\psi$ if there are differences between the male viabilities. For certain male and female viabilities polymorphisms can either be extinguished or generated by having worker produced males. The approach to linkage equilibrium at two loci is either accelerated or retarded, though by not very much, depending on the value of $\psi$, and the recombination rate.
\end{abstract}

\section{INTRODUCTION}

IN social Hymenoptera colonies are usually founded by a single fertilised queen who lays all of the female eggs and a certain proportion of the male eggs. In the honeybee virtually all of the males come from the queen as is the case with many ant species (Oster and Wilson, 1978). However, in many eusocial insects the workers have fully functioning ovaries and contribute a considerable proportion of the males. Contel and Kerr (1976) using genetic evidence estimated that 39 per cent of the males were sons of workers in a population of the stingless bee Melipona subnitida. Mixed male parentage is also common in wasps. Montagner found that there was a great variability between Vespula colonies; in some the queen was responsible for all of the males but in others she produced none, while in about half of the colonies examined the workers produced 75 per cent of the males (Spradbery, 1973). Worker oviposition commonly occurs in bumble bees; Zucchi estimates that in Bombus atratus workers lay 90 per cent of the male eggs (Michener, 1974 ), and in $B$. melanopygus genetic evidence indicates that the workers are the parents of about 40 per cent of the males though there is considerable intercolony variability (R. C. Plowright and R. E. Owen, unpublished data).

Hamilton's (1964) kin selection theory predicts that workers will be selected to raise their own sons, and this has stimulated collection of data on this question and a good deal of theoretical work on the consequences of this for the evolution of the social insects (e.g., Trivers and Hare, 1976; Oster, Eshel and Cohen, 1977).

However, the effect that worker produced males have on the population genetics of the social Hymenoptera has remained essentially unexplored. 
Hymenoptera have a haplo-diploid genetic system and so their population genetics is, in general, the same as for sex-linked genes in diplo-diploids. It differs on account of the absence of interactions between sex-linked and autosomal loci in the male haploids (Crozier, 1977) and because the biology permits such phenomena as worker produced males which have no counterpart in sex-linked genetics of diplo-diploid species.

In this paper only deterministic theory will be employed because the assumptions of a large panmictic population are quite well realised in the social Hymenoptera. Analysis will be confined to discrete non-overlapping generations. This is a realistic assumption for wasps (Vespinae, Polistinae), bumble bees (genus Bombus) and honeybees (Apis) of the temperate zones. In the tropics where breeding is less seasonal overlapping generations occur and the exact results will not apply; however, the conclusions as regards the directions of gene frequency change etc. caused by having worker produced males will still be valid. The object is to present the effects of the mixed origin of males on some aspects of hymenopteran population genetics.

\section{APPROACH TO HARDY-WEINBERG EQUILIBRIUM}

Assume a gene locus $g$ with alleles $g_{1}$ and $g_{2}$ initially at different frequencies in the two sexes. Let a proportion $\psi$ of the males in a colony be produced by workers and $(1-\psi)$ by the queen. The gene frequencies in each generation are shown in fig. 1. The male gene frequency in the second generation is $P^{\prime}=(1-\psi) p+\frac{1}{2} \psi(p+P)$ and the female gene frequency is $p^{\prime}=\frac{1}{2}(p+P)$. Therefore $P^{\prime}-p^{\prime}=-\frac{1}{2}(1-\psi)(P-p)$. Thus the gene frequency

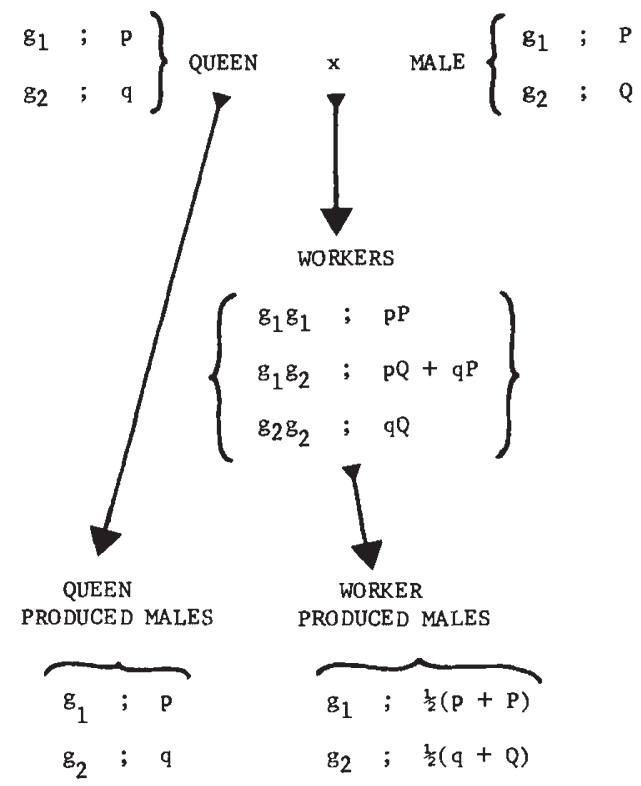

FIG. 1.-Gene frequencies after one generation of random mating if they are initially different in the two sexes and there is no selection. The worker's genotype frequencies are also shown. The second generation of young queens and the worker produced males have the same gene frequencies as the workers. 
difference between the sexes is reduced by the factor $\left[-\frac{1}{2}(1-\psi)\right]$ in each generation as opposed to $\left(-\frac{1}{2}\right)$, which applies in the usual diploid sex-linked case or when $\psi=0$. Similarly the difference in the $t$ th generation is given by

$$
P-p=\left(-\frac{1}{2}\right)^{t}(1-\psi)^{t}(P-p) \text {. }
$$

If $\psi=1$, then equilibrium is attained immediately in the next generation, as in the diploid autosomal case. The effect of a positive value of $\psi$ of any magnitude is to increase the speed of approach to equilibrium; though the gene frequency oscillations still occur at all values of $\psi$ except 1 . Note also that the relationship $(2-\psi) p^{\prime}+P^{\prime}=(2-\psi) p+P$ holds, $i . e$, , the quantity $(2-\psi) p+P$ is conserved from generation to generation. This is of significance in section 5 .

\section{EQUILIBRIUM BETWEEN MUTATION AND SELECTION}

Assume that the gene $g_{2}$ mutates to $g_{1}$ at rate $\mu$ per gene per generation. Back mutation is neglected. Suppose that $g_{1}$ is heterozygously expressed. Let the relative viabilities be

$$
\text { †๐ } \begin{array}{cccccc}
g_{1} g_{1} & g_{1} g_{2} & g_{2} g_{2} & \text { ठठ } & g_{1} & g_{2} \\
1-S_{11} & 1-S_{12} & 1 & & 1-S_{1} & 1
\end{array}
$$

where $0 \leqq S_{11}, S_{12} \leqq 1$, and $0 \leqq S_{1} \leqq 1$.

Then the genotypic frequencies after selection are

$$
\begin{aligned}
& \begin{array}{llll}
g_{1} g_{1} & g_{1} g_{2} & g_{2} g_{2}
\end{array} \\
& \left(1-S_{11}\right) p P / W \quad\left(1-S_{12}\right)(p Q+q P) / W \quad q Q / W
\end{aligned}
$$

where $W=1-S_{12}(p+P)-\left(S_{11}-2 S_{12}\right) p P$;

ठ઼

$$
\begin{array}{cc}
g_{1} & g_{2} \\
\frac{\left(1-S_{1}\right)\left[(1-\psi) p+\frac{1}{2} \psi(p+P)\right]}{1-S_{1}\left[(1-\psi) p+\frac{1}{2} \psi(p+P)\right]} & \frac{(1-\psi) q+\frac{1}{2} \psi(q+Q)}{1-S_{1}\left[(1-\psi) p+\frac{1}{2} \psi(p+P)\right]}
\end{array}
$$

The effect of mutation is to multiply the frequencies of the $g_{2}$ gene by $(1-\mu)$, thus if mutation and extinction are in balance, then in the females the gene $g_{2}$ is at frequency

$$
q=\frac{(1-\mu)\left[\frac{1}{2}\left(1-S_{12}\right)(p Q+q P)+q Q\right]}{1-S_{12}(p-P)-\left(S_{11}-2 S_{12}\right) p P},
$$

while its frequency in the males is

$$
Q=\frac{(1-\mu)\left[(1-\psi) q+\frac{1}{2} \psi(q+Q)\right]}{1-S_{1}\left[(1-\psi) p+\frac{1}{2} \psi(p+P)\right]} .
$$

Writing $q=1-p, Q=1-P$, and neglecting terms of the order of $p^{2}, P^{2}$ and $p P$, i.e., terms of order $\mu^{2}$, we obtain from equation (1) the simplified relation

$$
P=\left(p+S_{12} p-2 \mu\right) /\left(1-S_{12}\right) .
$$

Similarly from equation (2) we obtain

$$
1-S_{1}\left(p-\frac{1}{2} \psi p\right)=1-p+\frac{1}{2} \psi p-\mu+P\left(\frac{1}{2} \psi S_{1}-\frac{1}{2} \psi+1\right) .
$$


Substituting (3) into (4) and rearranging gives the equilibrium value for

$$
p=\frac{3 \mu-\mu\left[S_{12}+\psi\left(1-S_{1}\right)\right]}{S_{1}+2 S_{12}-S_{1} S_{12}-\psi S_{12}} .
$$

Substituting (5) in (3) gives the male equilibrium frequency

$$
P=\frac{3 \mu\left(1+S_{12}\right)-\mu\left[S_{12}+\psi\left(1-S_{1}\right)\right]\left(1+S_{12}\right)}{\left(1-S_{12}\right)\left(S_{1}+2 S_{12}-S_{1} S_{12}-\psi S_{12}\right)}-\frac{2 \mu}{\left(1-S_{12}\right)} .
$$

The effect of $\psi$ is not immediately obvious. However, differentiating $p$ with respect to $\psi$ shows that $d p / d \psi$ has the same sign as the expression $\left(3-S_{12}\right) S_{12}-\left(1-S_{1}\right)\left(S_{1}+2 S_{12}-S_{1} S_{12}\right)$, and is therefore positive provided that $S_{12}^{2}-\left(1+3 S_{1}-S_{1}^{2}\right) S_{12}+S_{1}\left(1-S_{1}\right)$ is negative, which is the case if $S_{1}>S_{0}$, where

$$
2 S_{0}=\left(1+3 S_{1}-S_{1}^{2}\right)-\sqrt{\left(1+3 S_{1}+S_{1}^{2}\right)^{2}-\psi S_{1}\left(1-S_{1}\right)} .
$$

Thus the effect of worker produced males is to increase $p$ and also $P$, when $S>S_{0}$. If $S_{1}=S_{0}$ then the equilibrium gene frequencies are independent of the value of $\psi$, and if $S_{1}<S_{0}$ then they decrease as $\psi$ increases.

If $g_{1}$ is recessive in females, then $S_{12}=0$ and the equilibrium frequencies (5) and (6) become $p=\left[3 \mu-\psi\left(1-S_{1}\right) \mu\right] / S_{1}$ and $P=p-2 \mu$. If $\psi=0$, then $p=3 \mu / S_{1}$ which is the standard result (Haldane, 1935) and $P=$ $\left(3 \mu / S_{1}\right)-2 \mu$. The frequencies $P$ and $p$ differ because they are the gene frequencies in the adults. This asymmetry between the sexes is not usually noted in sex-linked theory because they are not considered separately. In this case $\psi$ decreases $p$ and $P$, and thus it decreases the genetic load on the population. Taking $P=p$, the load based on the loss of individuals is

$$
\begin{aligned}
L & =\frac{1}{2}\left(S_{1} p+S_{11} P^{2}\right) \\
& \simeq 1 \cdot 5 \mu-\frac{1}{2} \psi\left(1-S_{1}\right) \mu,
\end{aligned}
$$

and based on the loss of the genetic material it is

$$
L=\frac{1}{3}\left(S_{1} p+2 S_{11} p^{2}\right) \simeq \mu-\frac{1}{3} \psi\left(1-S_{1}\right) \mu .
$$

When $\psi=0$ these equations reduce to the standard forms as given by Crozier (1976).

For the case where the $g_{1}$ gene is recessive and only expressed in the females it can be shown that

$$
P \simeq \sqrt{\left(\frac{3 \mu}{2 S_{11}}\right)\left(\frac{2-\psi}{2+\psi}\right)}
$$

and the effect of increasing the value of $\psi$ is to reduce the equilibrium frequency of $p$.

\section{Elimination of Deleterious alleles}

We consider the most general case of an unconditionally disadvantageous gene with viabilities $g_{1} g_{1}, 1-S_{11} ; g_{1} g_{2}, 1-S_{12} ; g_{2} g_{2}, 1 ; g_{1}$, 
$1-S_{1} ; g_{2}, 1$. The gene frequencies after one generation of selection are:

$$
\begin{aligned}
& p^{\prime}=\frac{\left(1-S_{11}\right) p P+\left(1-S_{12}\right)\left[\frac{1}{2}(p+P)-p P\right]}{1-S_{12}(p+P)-\left(S_{11}-2 S_{12}\right) p P}, \\
& P^{\prime}=\frac{\left(1-S_{1}\right)\left[(1-\psi) p+\frac{1}{2} \psi(p+P)\right]}{1-S_{1}\left[(1-\psi) p+\frac{1}{2} \psi(p+P)\right]}
\end{aligned}
$$

The rate of extinction of $g_{1}$ is determined to a high degree of accuracy by the equations resulting when second order terms in $p$ and $P$ (i.e., squares and products) are neglected. Since $S_{11}$ disappears when the product $p P$ is omitted the rate of extinction effectively depends only on the heterozygous and hemizygous disadvantages $S_{12}$ and $S_{1}$. Dropping the second order terms we have

$$
\begin{aligned}
& p^{\prime}=\frac{1}{2}\left(1-S_{12}\right)(p+P) \\
& P^{\prime}=\left(1-S_{1}\right)\left[\left(1-\frac{1}{2} \psi\right) p+\frac{1}{2} \psi P\right] .
\end{aligned}
$$

Solving the eigenvalue equation

$$
\left|\begin{array}{cc}
\frac{1}{2}\left(1-S_{12}\right)-\lambda & \frac{1}{2}\left(1-S_{12}\right) \\
\left(1-S_{1}\right)\left(1-\frac{1}{2} \psi\right) & \frac{1}{2} \psi\left(1-S_{1}\right)-\lambda
\end{array}\right|=0,
$$

we obtain the roots. The process of elimination of $g_{1}$ is governed by the larger root $\lambda_{1}$, where

$$
\begin{aligned}
4 \lambda_{1}= & \left(1-S_{12}\right)+\psi\left(1-S_{1}\right) \\
& +\sqrt{\left[\left(1-S_{12}\right)+\psi\left(1-S_{1}\right)\right]^{2}+8\left(1-S_{12}\right)\left(1-S_{1}\right)(1-\psi)} .
\end{aligned}
$$

The smaller root $\lambda_{2}$ is given by changing the sign of the radical. To ascertain the effect of having worker produced males let $\kappa=\left(1-S_{12}\right) /\left(1-S_{1}\right)$. The cases $S_{1}<S_{12}, S_{1}=S_{12}, S_{1}>S_{12}$ then correspond respectively to $\kappa<1, \kappa=1$ and $\kappa>1$. We find that $4 \lambda_{1} /\left(1-S_{1}\right)=\kappa+\psi+\sqrt{\left[(3-\psi)^{2}+8 \kappa(1-\kappa)\right]}$, it follows that if $S_{1}=S_{12}$ and $\kappa=1$, then $\lambda_{1}$ is independent of $\psi$, being simply equal to the relative viability $\left(1-S_{1}\right)$. Otherwise

$$
\frac{4}{\left(1-S_{1}\right)} \frac{d \lambda_{1}}{d \psi}=1+\frac{(\psi-3 \kappa)}{\sqrt{\left[(\psi-3 \kappa)^{2}+8 \kappa(1-\kappa)\right]}} .
$$

If $S_{1}<S_{12}$, then $d \lambda_{1} / d \psi$ is positive because the second term above is positive if $\kappa \leqq \frac{1}{3} \psi$, and if $\frac{1}{3} \psi<\kappa<1$ it is negative but less than unity. Hence if $S_{1}<S_{12}$ (i.e., when $g_{1}$ is relatively less disadvantageous in the males than in the females) then $\lambda_{1}$ increases with increasing values of $\psi$. That is, having worker produced males reduces the rate of elimination of a deleterious allele. If $S_{1}>S_{12}$ then $d \lambda_{1} / d \psi$ is negative because the second term above is negative and exceeds unity. Thus $\lambda_{1}$ diminishes as $\psi$ increases, i.e., the presence of worker males increases the rate of elimination of a gene which is relatively more disadvantageous in the males than in the females, as expected intuitively.

\section{The SPREAD of AN ADVANTAgeous Allele}

In this section we will investigate the effect of worker produced males on the spread of an advantageous allele that has a small additive effect on 
fitness. We seek an expression to describe the rate of change of gene frequency which is analogous to that of the autosomal diploid case, $d p / d t \simeq$ $2 p q \alpha$ (Fisher, 1930), where $\alpha$ is the average effect of the allele. Unlike Hartl (1972) we do not assume dosage compensation (i.e., that the fitness of each hemizygous male is identical to that of the corresponding female) nor that the gene frequencies in each sex are equal.

For five genotypes the relative viabilities are

or

ๆ९ $\begin{array}{cccccc}g_{1} g_{1} & g_{1} g_{2} & g_{2} g_{2} & \text { ठठ } & g_{1} & g_{2} \\ w_{11} & w_{12} & w_{22} & & v_{1} & v_{2} \\ 1+m_{11} & 1+m_{12} & 1+m_{22} & & 1+m_{1} & 1+m_{2} .\end{array}$

Considering slow selection only, we obtain an expression for changes in gene frequencies in terms of fitness values. Thus for the females

$$
\begin{aligned}
& W p^{\prime}=\frac{1}{2}(p+P)+m_{11} p P+\frac{1}{2} m_{12}(p Q+q P) \\
& W q^{\prime}=\frac{1}{2}(q+Q) \quad+\frac{1}{2} m_{12}(p Q+q P)+m_{22} q Q
\end{aligned}
$$

with

$$
\begin{aligned}
W & =1+\left(m_{11} p+m_{12} q\right) P+\left(m_{12} p+m_{22} q\right) Q \\
& =1+T-\alpha(p-P)
\end{aligned}
$$

where

$$
T=m_{11} p^{2}+2 m_{12} p q+m_{22} q^{2}
$$

and

$$
\alpha=\left(m_{11}-m_{12}\right) p+\left(m_{12}-m_{22}\right) q .
$$

Subtracting (8) from (7) gives

$$
\begin{aligned}
W\left(p^{\prime}-q^{\prime}\right) & =(p-q)-(p-P)+m_{11} p P-m_{22} q Q \\
& =(p-q)-(p-P)+m_{11} p^{2}-m_{22} q^{2}-\left(m_{11} p+m_{22} q\right)(p-P) .
\end{aligned}
$$

Therefore, because

$$
\begin{aligned}
1 / W & =1 /[1+T-\alpha(p-P)] \\
& =1-T+\alpha(p-P)+O\left(m^{2}\right),
\end{aligned}
$$

where $O\left(\mathrm{~m}^{2}\right)$ denotes terms which are of the second degree in $m$ and so are ignored under the hypothesis of slow selection, (9) reduces to

$$
p^{\prime}-q^{\prime}=(p-q)-(p-P)+2 p q \alpha+\phi(p-P)-\alpha(p-P)^{2}+O\left(m^{2}\right),
$$

where $\phi=T+\alpha(p-q)-\left(m_{11} p+m_{22} q\right)$. Because $q=1-p, q^{\prime}=1-p^{\prime}$ we have

$$
p^{\prime}-p=p q \alpha-\frac{1}{2}(p-P)+\frac{1}{2} \phi(p-P)-\frac{1}{2} \alpha(p-P)^{2}+O\left(m^{2}\right) .
$$

Similarly for the males we have

$$
\begin{aligned}
V P^{\prime} & =(1-\psi)\left(1+m_{1}\right) p+\frac{1}{2} \psi\left(1+m_{1}\right)(P+p), \\
V Q^{\prime} & =(1-\psi)\left(1+m_{2}\right) q+\frac{1}{2} \psi\left(1+m_{2}\right)(Q+q),
\end{aligned}
$$


or

$$
\begin{gathered}
V P^{\prime}=p+m_{1} p-\frac{1}{2} \psi(p-P)\left(1+m_{1}\right), \\
V Q^{\prime}=q+m_{2} q-\frac{1}{2} \psi(q-Q)\left(1+m_{2}\right),
\end{gathered}
$$

where

$$
V=1+m_{1} p+m_{2} q+\frac{1}{2} \psi\left(m_{2}-m_{1}\right)(p-P) .
$$

Therefore, subtracting (12) from (11) gives

$$
V\left(P^{\prime}-Q^{\prime}\right)=(p-q)+\left(m_{1} p-m_{2} q\right)-\frac{1}{2} \psi\left(2+m_{1}+m_{2}\right)(p-P) .
$$

Multiplying (13) by $1 / V$, we obtain

$$
P^{\prime}-Q^{\prime}=p-q+2 p q \beta-\psi(p-P)-\psi\left(m_{1}+m_{2}\right)(p-P)+O\left(m^{2}\right),
$$

where $\beta=m_{1}-m_{2}$. Substituting $2 P^{\prime}-1$ for $P^{\prime}-Q^{\prime}$ in (14) gives

$$
P^{\prime}=p-p q \beta-\frac{1}{2} \psi(p-P)-\frac{1}{2} \psi\left(m_{1}+m_{2}\right)(p-P)+O\left(m^{2}\right),
$$

and because $p=P+(p-P)$, it follows that

$$
P^{\prime}-P=p q \beta+(p-P)-\frac{1}{2} \psi(p-P)-\frac{1}{2} \psi\left(m_{1}+m_{2}\right)(p-P)+O\left(m^{2}\right) .
$$

Note that $\alpha$ and $\beta$ are equal to the $\beta$ and $\gamma$ respectively of Hartl (1971, 1972). Adding the gene frequency changes in females, equation (10), and males, equation (15), and weighing the contribution of the sexes appropriately, gives the overall effect:

$$
\begin{aligned}
2\left(p^{\prime}-p\right)+\left(P^{\prime}-P\right)= & (2 \alpha+\beta) p q-\frac{1}{2} \psi(p-P) \\
& +\phi(p-P)-\alpha(p-P)^{2}+O\left(m^{2}\right) .
\end{aligned}
$$

Thus worker produced males influence the rate of change of the "overall gene frequency" $(2 p+P)$ only when there is frequency disequilibrium between the sexes, i.e., $p$ and $P$ are unequal. This is because the term $\frac{1}{2} \psi(p-P)$ in equation (16) equals 0 when $p=P$.

We noted in section 2 that the quantity $(2-\psi) p+P$ is conserved in the absence of selection. Under the present assumption of slow selection we find that

$$
\begin{aligned}
{\left[(2-\psi) p^{\prime}+P^{\prime}\right]-[(2-\psi) p+P]=} & (2-\psi)\left(p^{\prime}-p\right)+\left(P^{\prime}-P\right) \\
= & {[(2-\psi) \alpha+\beta] p q+O(m)(p-P) } \\
& +O(m)(p-P)^{2}+O\left(m^{2}\right) .
\end{aligned}
$$

Thus, even when $p=P$, the "weighted gene frequency" $(2-\psi) p+P$ is not conserved but changes at the approximate rate $[(2-\psi) \alpha+\beta] p q$ per generation. If $\alpha$ and $\beta$ are both positive so that $g_{1}$ is advantageous in either sex, then $(2-\psi) p+P$ increases. The quantity $(2-\psi) \alpha+\beta$ may be thought of as the "weighted advantage" of $g_{1}$ the advantageous allele. We may expect that after a sufficiently long initial period the disequilibrium $(p-P)$ becomes, and remains, of the order $m$, in which case the increase per generation is

$$
[(2-\psi) \alpha+\beta] p q+O\left(m^{2}\right)
$$

Therefore worker produced males actually slow the rate of increase of an advantageous allele having additive effects and under slow selection. 


\section{POLYMORPHISMS AT A SINGLE LOCUS}

Assume viabilities

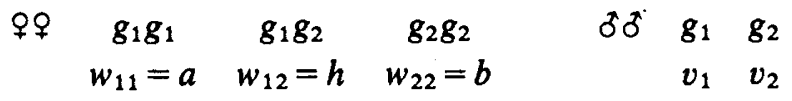

with gene frequencies $\$: g_{1}, p ; g_{2}, q ; \delta: g_{1}, P ; g_{2}, Q$. Let $p_{e}, q_{e} ; P_{e}, Q_{e}$ and $W_{e}, V_{e}$ denote equilibrium values where $W_{e}$ and $V_{e}$ are the mean viabilities given respectively by

$$
\begin{aligned}
W_{e} & =\left(a P_{e}+h Q_{e}\right) p_{e}+\left(h P_{e}+Q_{e}\right) q_{e}, \\
V_{e} & =\left(1-\frac{1}{2} \psi\right)\left(v_{1} p_{e}+v_{2} q_{e}\right)+\frac{1}{2} \psi\left(v_{1} P_{e}+v_{2} Q_{e}\right) .
\end{aligned}
$$

Then in a polymorphic equilibrium,

$$
\begin{array}{ll}
W_{e} p_{e}=a p_{e} P_{e}+\frac{1}{2} h\left(p_{e} Q_{e}+q_{e} P_{e}\right), \\
W_{e} q_{e}= & \frac{1}{2} h\left(p_{e} Q_{e}+q_{e} P_{e}\right)+b q_{e} Q_{e},
\end{array}
$$

and

$$
\begin{aligned}
V_{e} P_{e} & =v_{1}\left[\left(1-\frac{1}{2} \psi\right) p_{e}+\frac{1}{2} P_{e}\right] \\
V_{e} Q_{e} & =v_{2}\left[\left(1-\frac{1}{2} \psi\right) q_{e}+\frac{1}{2} Q_{e}\right]
\end{aligned}
$$

or by rearrangement

$$
\begin{array}{r}
P_{e}=\frac{\left(1-\frac{1}{2} \psi\right) v_{1} p_{e}}{V_{e}-\frac{1}{2} \psi v_{1}}, \\
Q_{e}=\frac{\left(1-\frac{1}{2} \psi\right) v_{2} q_{e}}{V_{e}-\frac{1}{2} \psi v_{2}}
\end{array}
$$

Therefore, substituting equations (18) into (17) we get

$$
\begin{array}{ll}
\frac{W_{e} p_{e}}{\left(1-\frac{1}{2} \psi\right)}=\frac{a p_{e} v_{1} p_{e}}{V_{e}-\frac{1}{2} \psi v_{1}}+\frac{\frac{1}{2} h v_{2} p_{e} q_{e}}{V_{e}-\frac{1}{2} \psi v_{2}}+\frac{\frac{1}{2} h v_{1} p_{e} q_{e}}{V_{e}-\frac{1}{2} \psi} \\
\frac{W_{e} q_{e}}{\left(1-\frac{1}{2} \psi\right)}= & \frac{\frac{1}{2} h v_{2} p_{e} q_{e}}{V_{e}-\frac{1}{2} \psi v_{2}}+\frac{\frac{1}{2} h v_{1} p_{e} q_{e}}{V_{e}-\frac{1}{2} \psi v_{1}}+\frac{b q_{e} v_{2} q_{e}}{V_{e}-\frac{1}{2} \psi v_{2}}
\end{array}
$$

which are equivalent to

$$
V_{e} W_{e} p_{e}=\left(A^{*} p_{e}+H^{*} q_{e}\right) p_{e}, \quad V_{e} W_{e} q_{e}=\left(H^{*} p_{e}+B^{*} q_{e}\right) q_{e},
$$

where

$$
\begin{gathered}
A^{*}=\frac{\left(1-\frac{1}{2} \psi\right) V_{e}}{V_{e}-\frac{1}{2} \psi v_{1}} a v_{1}, \quad B^{*}=\frac{\left(1-\frac{1}{2} \psi\right) V_{e}}{V_{e}-\frac{1}{2} \psi v_{2}} b v_{2} \\
\left.H^{*}=\frac{\left(1-\frac{1}{2} \psi\right) V_{e}}{V_{e}-\frac{1}{2} \psi v_{1}}\left(\frac{1}{2} h v_{1}\right)+\frac{\left(1-\frac{1}{2} \psi\right) V_{e}}{V_{e}-\frac{1}{2} \psi v_{2}} h v_{2}\right)
\end{gathered}
$$


It follows that

$$
\begin{aligned}
& p_{e}=\frac{H^{*}-B^{*}}{2 H^{*}-A^{*}-B^{*}}, \\
& q_{e}=\frac{H^{*}-A^{*}}{2 H^{*}-A^{*}-B^{*}} .
\end{aligned}
$$

If $\psi=0$, then $A^{*}=a, B^{*}=b$ and $H^{*}=\frac{1}{2} h\left(v_{1}+v_{2}\right)$. Since $a=w_{11}, h=w_{12}$, $b=w_{22}$ the equilibrium gene frequency equations reduce to their standard form as given in Edwards (1977). We also note that in the general case

$$
\begin{aligned}
V_{e} W_{e} & =A^{*} p_{e}+H^{*} q_{e}+H^{*} p_{e}+B^{*} q_{e} \\
& =\left(H^{*}-A^{*} B^{*}\right) /\left(2 H^{*}-A^{*}-B^{*}\right) .
\end{aligned}
$$

To obtain an expression for $V_{e}$ we see that

$$
\begin{aligned}
\frac{2 H^{*}-A^{*}-B^{*}}{\left(1-\frac{1}{2} \psi\right)}= & \frac{\left(2 H^{*}-A^{*}-B^{*}\right)\left(P_{e}-Q_{e}\right)}{\left(1-\frac{1}{2} \psi\right)}=\frac{\left(1-\frac{1}{2} \psi\right) V_{e} \frac{1}{2} h v_{1}^{2}}{\left(V_{e}-\frac{1}{2} \psi v_{1}\right)^{2}} \\
& +\frac{\left(1-\frac{1}{2} \psi\right) V_{e}(h-a-b) v_{1} v_{2}}{\left(V_{e}-\frac{1}{2} \psi v_{1}\right)\left(V_{e}-\frac{1}{2} \psi v_{2}\right)}+\frac{\left(1-\frac{1}{2} \psi\right) V_{e} \frac{1}{2} h v_{2}^{2}}{\left(V_{e}-\frac{1}{2} \psi v_{2}\right)^{2}} .
\end{aligned}
$$

The left hand side of the equation is also equal to

$$
\frac{v_{1}(h-a) V_{e}}{V_{e}-\frac{1}{2} \psi v_{1}}+\frac{v_{2}(h-b) V_{e}}{V_{e}-\frac{1}{2} \psi v_{2}},
$$

so by equating and rearranging, we get

$$
\begin{aligned}
\frac{v_{1}(h-a)}{V_{e}-\frac{1}{2} \psi v_{1}}+\frac{v_{2}(h-b)}{V_{e}-\frac{1}{2} \psi v_{2}}= & \left(1-\frac{1}{2} \psi\right)\left[\frac{\frac{1}{2} h_{1} v_{1}^{2}}{\left(V_{e}-\frac{1}{2} \psi v_{1}\right)^{2}}\right. \\
& \left.+\frac{(h-a-b) v_{1} v_{2}}{\left(V_{e}-\frac{1}{2} \psi v_{1}\right)\left(V_{e}-\frac{1}{2} \psi v_{2}\right)}+\frac{\frac{1}{2} h v_{2}^{2}}{\left(V_{e}-\frac{1}{2} \psi v_{2}\right)^{2}}\right]
\end{aligned}
$$

Equation (21) can be put in the form of $f\left(V_{e}\right)=0$, where

$$
\begin{aligned}
f\left(V_{e}\right)= & \left(V_{e}-\frac{1}{2} \psi v_{1}\right)\left(V_{e}-\frac{1}{2} \psi v_{2}\right)\left[v _ { 1 } ( h - a ) \left(V_{e}-\frac{1}{2} \psi v_{2}\right.\right. \\
& \left.+v_{2}(h-b)\left(V_{e}-\frac{1}{2} \psi v_{1}\right)\right]-\left(1-\frac{1}{2} \psi\right)\left[\frac{1}{2} h v_{1}^{2}\left(V_{e}-\frac{1}{2} \psi v_{2}\right)^{2}\right. \\
& \left.+(h-a-b) v_{1} v_{2}\left(V_{e}-\frac{1}{2} \psi v_{1}\right)\left(V_{e}-\frac{1}{2} \psi v_{2}\right)+\frac{1}{2} h v_{2}^{2}\left(V_{e}-\frac{1}{2} \psi v_{1}\right)^{2}\right] .
\end{aligned}
$$

For the polymorphism to be stable, both roots of the quadratic equation $V_{e} W_{e} \lambda^{2}-\left(V_{e} R+\frac{1}{2} \psi W_{e} U\right) \lambda-\left[S U-\frac{1}{2} \psi(R+S) U\right]=0$ must be less than unity in absolute value, where

$$
\begin{aligned}
R & =\left(a P_{e}+\frac{1}{2} h Q_{e}-\frac{1}{2} h P_{e}\right) q_{e}-\left(\frac{1}{2} h Q_{e}+\frac{1}{2} h P_{e}-b Q_{e}\right) P_{e}, \\
S & =(a-2 h+b) p_{e} q_{e}+\frac{1}{2} h, \\
U & =v_{1} Q_{e}+v P_{e} .
\end{aligned}
$$


A necessary and sufficient set of conditions for stability is that the "stability coefficient",

$$
\frac{\left(V_{e} R+S U\right)+\frac{1}{2} U\left[W_{e}-(R+S)\right]}{V_{e} W_{e}}
$$

is positive and less than unity. The larger the value of the coefficient, up to unity, the longer it takes the system to return to equilibrium after a perturbation. Proof of this and a full analytical solution of the polymorphism equations will be given elsewhere (A. R. G. Owen and R. E. Owen, in preparation). For the purpose of this paper there is enough information to be able to investigate the effect of mixed male parentage on polymorphisms. To do this, numerical examples were worked out. First viability values $a, h, b$, and $v_{1}, v_{2}$ were chosen, then an estimate of $V_{e}$ was calculated which gave $f\left(V_{e}\right)=0$, (equation 22) for each of eleven values of $\psi$. Equations (18), (19) and (20) were then used to calculate the equilibrium gene frequencies. The stability coefficient equation (23) was also calculated. The numerical examples are shown in table 1 and they illustrate the major effects that worker produced males have on polymorphism. Example (i) shows that even for modest values of $\psi$ the equilibrium gene frequencies are shifted and in the direction, not surprisingly, of the male genotype with the greater viability. The system also remains stable. For a given set of female viabilities the shift in gene frequencies depends on the magnitude of the

TABLE 1

Numerical examples illustrating the effect of worker produced males on the equilibrium gene frequencies and stability of balanced polymorphisms. If the stability coefficient (calculated from equation 23 ) is less than 1.0 then the polymorphism is stable

\begin{tabular}{|c|c|c|c|c|c|c|c|c|c|c|c|c|}
\hline \multirow[b]{2}{*}{ Viabilities } & \multirow{2}{*}{$\begin{array}{l}\text { Gene } \\
\text { freqs. }\end{array}$} & & & & & & \\
\hline & & $0 \cdot 0$ & $0 \cdot 1$ & $0 \cdot 2$ & $0 \cdot 3$ & 0.4 & $0 \cdot 5$ & 0.6 & 0.7 & $0 \cdot 8$ & 0.9 & $1 \cdot 0$ \\
\hline \multicolumn{13}{|l|}{ (i) } \\
\hline$h=10 \cdot 0$ & ㅇ $p$ & 0.750 & 0.758 & $0 \cdot 766$ & 0.774 & 0.782 & 0.791 & 0.800 & 0.810 & 0.820 & 0.830 & 0.841 \\
\hline$a=5 \cdot 0$ & $q$ & 0.250 & $0 \cdot 242$ & $0 \cdot 234$ & $0 \cdot 226$ & 0.218 & $0 \cdot 209$ & 0.200 & 0.190 & $0 \cdot 180$ & $0 \cdot 170$ & $0 \cdot 159$ \\
\hline$b=5 \cdot 0$ & $\sigma P$ & 0.900 & 0.905 & 0.914 & 0.921 & 0.928 & 0.935 & 0.942 & 0.948 & 0.954 & 0.960 & 0.965 \\
\hline$v_{1}=3 \cdot 0$ & $Q$ & $0 \cdot 100$ & 0.095 & 0.086 & 0.079 & 0.072 & 0.065 & 0.058 & 0.052 & 0.046 & 0.040 & 0.035 \\
\hline$v_{2}=1 \cdot 0$ & Stability & 0.769 & 0.779 & 0.788 & 0.798 & 0.808 & 0.818 & 0.828 & 0.837 & 0.848 & 0.858 & 0.868 \\
\hline \multicolumn{13}{|l|}{ (ii) } \\
\hline$h=10 \cdot 0$ & 우 $p$ & 0.517 & $0 \cdot 518$ & 0.519 & 0.520 & 0.522 & 0.523 & 0.525 & 0.526 & 0.529 & 0.531 & 0.534 \\
\hline$a=5 \cdot 0$ & $q$ & 0.483 & 0.482 & 0.481 & 0.480 & 0.487 & 0.477 & 0.475 & 0.474 & 0.471 & 0.469 & 0.466 \\
\hline$b=5 \cdot 0$ & đ P & 0.534 & 0.535 & 0.537 & 0.540 & 0.543 & 0.546 & 0.549 & 0.553 & 0.557 & 0.562 & 0.573 \\
\hline$v_{1}=3 \cdot 0$ & $Q$ & 0.466 & 0.465 & 0.463 & 0.460 & 0.457 & 0.454 & 0.451 & 0.447 & 0.443 & 0.438 & 0.427 \\
\hline$v_{2}=2 \cdot 8$ & Stability & 0.667 & 0.683 & $0 \cdot 700$ & 0.717 & 0.734 & 0.750 & 0.767 & 0.783 & 0.800 & 0.816 & 0.837 \\
\hline \multicolumn{13}{|c|}{ 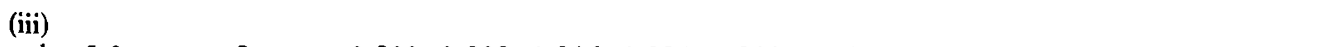 } \\
\hline$h=5 \cdot 0$ & 우 $p$ & 0.900 & 0.908 & 0.916 & 0.924 & 0.933 & 0.943 & 0.953 & 0.963 & 0.975 & 0.987 & $1 \cdot 000$ \\
\hline$a=3 \cdot 0$ & $q$ & $0 \cdot 100$ & 0.092 & 0.084 & 0.076 & 0.067 & 0.057 & 0.047 & 0.037 & 0.025 & 0.013 & 0.000 \\
\hline$b=1 \cdot 0$ & $\sigma P$ & 0.964 & 0.968 & 0.972 & 0.976 & 0.980 & 0.984 & 0.988 & 0.991 & 0.994 & 0.997 & $1 \cdot 000$ \\
\hline$v_{1}=3.0$ & $Q$ & 0.036 & 0.032 & 0.028 & 0.024 & 0.020 & 0.016 & 0.012 & 0.009 & 0.006 & 0.003 & 0.000 \\
\hline$v_{2}=1 \cdot 0$ & Stability & 0.901 & 0.910 & 0.920 & 0.929 & 0.939 & 0.948 & 0.958 & 0.968 & 0.978 & 0.989 & $1 \cdot 000$ \\
\hline \multicolumn{13}{|l|}{ (iv) } \\
\hline$h=1.82$ & ㅇ $p$ & 0.000 & $0 \cdot 213$ & $0 \cdot 296$ & 0.353 & 0.397 & 0.433 & 0.464 & 0.491 & 0.511 & 0.536 & 0.555 \\
\hline$a=0.5$ & & $1 \cdot 000$ & 0.787 & $0 \cdot 704$ & 0.647 & 0.603 & 0.567 & 0.536 & 0.509 & 0.489 & 0.464 & 0.445 \\
\hline$b=10 \cdot 0$ & $\delta P$ & 0.000 & 0.760 & 0.844 & 0.884 & 0.909 & 0.926 & 0.939 & 0.950 & 0.922 & 0.967 & 0.970 \\
\hline$v_{1}=10 \cdot 0$ & $Q$ & $1 \cdot 000$ & $0 \cdot 240$ & $0 \cdot 156$ & 0.116 & 0.091 & 0.074 & $0 \cdot 061$ & 0.050 & 0.078 & 0.033 & 0.030 \\
\hline$v_{2}=1 \cdot 0$ & Stability & $1 \cdot 000$ & 0.833 & 0.767 & 0.725 & 0.695 & 0.673 & 0.655 & 0.642 & 0.622 & 0.622 & 0.614 \\
\hline
\end{tabular}


difference in viability between the males and illustrated by the comparison between examples (i) and (ii). Sufficient magnitude of $\psi$ can also cause a polymorphism to be extinguished: example (iii). For this to occur one of the alleles must be reasonably near fixation. The most interesting effect of having worker males is to cause stable polymorphisms to be generated from a non-polymorphic situation as shown in example (iv). With the viabilities chosen $A^{*}=5 \cdot 0, B^{*}=10 \cdot 0$ and $H^{*}=10 \cdot 0$, so that $p_{e}=P_{e}=0.00$ and $q_{e}=Q_{e}=1.00$ when $\psi=0$. However, because the viabilities are asymmetrical between the sexes $\left(a<b, v_{1}>v_{2}\right)$ a polymorphism results when $\psi>0$.

It is well-known that in situations like this, where the fitness of the corresponding hemizygotes and homozygotes differ in opposite directions, a stable polymorphism can result with $\psi=0$ even if the female heterozygote is less fit than the mean of the homozygotes (Bennet, 1957; Haldane and Jayakar, 1964; Li, 1967). However, as can be seen from example (iv) the conditions are relaxed when there are worker produced males. Thus to get a polymorphism when $\psi>0, v_{1}$ need only equal $b$ rather than be greater than $b$ which is required when $\psi=0$. Worker produced males have no effect on a polymorphism when there is no selection among the males $\left(v_{1}=v_{2}\right)$. In this case which is the same when $\psi=0$, equations (20) reduce to the standard diplo-diploid form and the gene frequencies are the same in the two sexes (Li, 1967). However, as can be seen from table 1, worker produced males have an effect whether there is complete dosage compensation $\left(v_{1}=a\right.$, $\left.v_{2}=b\right)$ or lack of it $\left(v_{1} \neq a, v_{2} \neq b\right)$. In fact it is a distinct possibility that the viabilities of the corresponding male and female genotypes could differ considerably since in many social hymenopteran groups the sexes have quite different life styles and may be exposed to different and often opposing selection pressures. For instance, queen bumble bees and wasps are physiologically adapted for hibernation during the winter while males live for only a few weeks at the end of the summer. Similarly, males are automimics of their sisters who are protected by their stings (Plowright and Owen, 1980) and are therefore selected to resemble them closely; but there also may exist opposing selective pressures on the same characters (hair length and colour, etc.) which work to reduce the resemblance (Stiles, 1979). Thus situations like example (iv) (table 1) may be quite likely. It is, of course, well-known that opposing selection in the two sexes of diplo-diploids can cause polymorphisms (Owen, 1953) and there are a number of examples (e.g., Turner, 1968).

\section{APPROACH TO EOUILIBRIUM AT TWO LOCI}

Assuming two loci with two alleles per locus denoted by $A_{1}, a_{1} ; A_{2}, a_{2}$, let the four types of gametes have frequencies

$\begin{array}{ccccc} & A_{1} A_{2} & a_{1} a_{2} & A_{1} a_{2} & a_{1} A_{2} \\ \text { 우 } & x_{1} & x_{2} & x_{3} & x_{4} \\ \text { ॐठ } & y_{1} & y_{2} & y_{3} & y_{4}\end{array}$

where $x_{1}+x_{2}+x_{3}+x_{4}=y_{1}+y_{2}+y_{3}+y_{4}=1$. The frequencies of the 16 offspring genotypes are given by the terms in the expansion of $\left(x_{1}+x_{2}+x_{3}+x_{4}\right)$ 
$\left(y_{1}+y_{2}+y_{3}+y_{4}\right)$. Calculating the gametic frequencies in the next generation with recombination rate $c$ we find that

and

$$
\begin{aligned}
& x_{1}^{\prime}=\frac{1}{2} x_{1}+\frac{1}{2} y_{1}-\frac{1}{2} c I, \\
& x_{2}^{\prime}=\frac{1}{2} x_{2}+\frac{1}{2} y_{2}-\frac{1}{2} c I, \\
& x_{3}^{\prime}=\frac{1}{2} x_{3}+\frac{1}{2} y_{3}+\frac{1}{2} c I, \\
& x_{4}^{\prime}=\frac{1}{2} x_{4}+\frac{1}{2} y_{4}+\frac{1}{2} c I,
\end{aligned}
$$

$$
\begin{aligned}
& y_{1}^{\prime}=(1-\psi) x_{1}+\psi x_{1}^{\prime}, \\
& y_{2}^{\prime}=(1-\psi) x_{2}+\psi x_{2}^{\prime}, \\
& y_{3}^{\prime}=(1-\psi) x_{3}+\psi x_{3}^{\prime}, \\
& y_{4}^{\prime}=(1-\psi) x_{4}+\psi x_{4}^{\prime} .
\end{aligned}
$$

In haplo-diploidy we have not only the coefficient of linkage disequilibrium $D=x_{1} x_{2}-x_{3} x_{4}$, but also $I=x_{1} y_{1}+x_{2} y_{1}-x_{3} y_{4}-x_{4} y_{3}$, the "coefficient of linkage-frequency disequilibrium", between the sexes. $I$ has a maximum value of 0.5 while $D$ has a maximum of 0.25 . If $x_{1}=y_{1}, x_{2}=y_{2}, x_{3}=y_{3}$, $x_{4}=y_{4}$ then $I=2 D$. We are interested in finding the rate at which both $D$ and $I$ tend to zero. To find the speed of approach to equilibrium we need to find and solve the recurrence relations for $I$ and $D$. Dealing with $I$ first,

$$
I^{\prime}=x_{1}^{\prime} y_{2}^{\prime}+x_{2}^{\prime} y_{1}^{\prime}-x_{3}^{\prime} y_{4}^{\prime}-x_{4}^{\prime} y_{3}^{\prime} \text {. }
$$

By substituting equations (24) and (25) in (26) we get

$$
I^{\prime}=\left(1-\frac{1}{2} \psi\right) D+\frac{1}{2} I-\frac{1}{2} c(1-\psi) I+\frac{1}{2} \psi\left(y_{1} y_{2}-y_{3} y_{4}\right) .
$$

Now calculating $\left(y_{1}^{\prime} y_{2}^{\prime}-y_{3}^{\prime} y_{4}^{\prime}\right)$ using equations (25) we obtain

$$
y_{1}^{\prime} y_{2}^{\prime}-y_{3}^{\prime} y_{4}^{\prime}=(1-\psi)^{2} D+\psi(1-\psi)\left(D+\frac{1}{4} I\right)+\psi^{2} D^{\prime} \text {. }
$$

Priming (27) and substituting (28) into it gives

$$
I^{\prime \prime}=\left(1-\frac{1}{2} \psi-\frac{1}{2} \psi^{3}\right) D^{\prime}+\frac{1}{2} \psi(1-\psi) D+\frac{1}{2} I^{\prime}-\frac{1}{2} c(1-\psi) I^{\prime}+\frac{1}{8} \psi^{2}(1-\psi) I
$$

Next we calculate $D^{\prime}$ from equations (24),

$$
x_{1}^{\prime} x_{2}^{\prime}-x_{3}^{\prime} x_{4}^{\prime}=\frac{1}{4}\left(x_{1} x_{2}-x_{3} x_{4}\right)+\frac{1}{4}\left(y_{1} y_{2}-y_{3} y_{4}\right)+\frac{1}{4} I^{\prime}-\frac{1}{2} c I^{\prime} .
$$

By priming equation (30) and substituting (28) into it we obtain

$$
D^{\prime \prime}=\frac{1}{4}\left(1-\psi^{2}\right) D^{\prime}+\frac{1}{4}(1-\psi) D+\frac{1}{4}(1-2 c) I^{\prime}+\frac{1}{16} \psi(1-\psi) I^{\prime} .
$$

We have, therefore, recurrence relations for $D$ and $I$ each of which involves both $D$ and $I$.

By successively priming and substituting equations (29) and (31) into each other $I$ can be eliminated to give a fourth-order equation in $D$ which has a corresponding eigenvalue equation:

$$
\begin{aligned}
\lambda^{4}- & {\left[\frac{3}{4}\left(1-\frac{1}{3} \psi^{2}\right)-\frac{1}{2} c(1-\psi)\right] \lambda^{3}-\left[\left(\frac{1}{4}-\frac{1}{2} c\right)\left(1-\frac{1}{2} \psi+\frac{1}{2} \psi^{3}\right)\right.} \\
& +\frac{1}{8} c(1-\psi)\left(1+\psi^{2}\right)+(1-\psi)\left(\frac{1}{4}+\frac{1}{16} \psi+\frac{3}{32} \psi^{2}+\frac{1}{32} \psi^{4}\right) \\
& \left.-\frac{1}{8}\left(1+\psi^{2}\right)\right] \lambda^{2}-\left[(1-\psi)\left\{\frac{1}{8} \psi(1-2 c)-\frac{1}{32} \psi^{2}\left(1+\psi^{2}\right)-\frac{1}{8}\right\}\right. \\
& \left.+\left(1-\psi^{2}\right)\left(\frac{1}{32} \psi^{2}+\frac{1}{8} c\right)\right] \lambda+\frac{1}{32} \psi^{2}(1-\psi)^{2}=0
\end{aligned}
$$


The roots of the equation give the speed of approach to linkage equilibrium. Specifically the value of $D$ in the $t$ th generation is $D_{t}=$ $k_{1} \lambda_{1}^{t}+k_{2} \lambda_{2}^{t}+k_{3} \lambda_{3}^{t}+k_{4} \lambda_{4}^{t}$ where $k_{1}, k_{2}, k_{3}, k_{4}$ are constants. If $\left|\lambda_{1}\right|>$ $\left|\lambda_{2}\right|,\left|\lambda_{3}\right|,\left|\lambda_{4}\right|$ then $D \simeq k_{1} \lambda_{1}^{t}$ when $t$ is large. That is, the largest root, $\lambda_{1}$, is the one that effectively determines the rate of approach to equilibrium when it takes more than a few generations to be reached. The smaller the value of $\lambda_{1}$ then the faster the approach to equilibrium.

Both $D$ and $I$ decrease at the same rate. For comparison the corresponding result for the autosomal diplo-diploid case is, as is well-known, $D_{t}=D_{0}(1-c)^{t}$, where the constant $D_{0}$ is the initial value of $D$. The constant $k_{1}$ is more complicated because it depends on $D_{0}, I_{0}$ and the $\lambda$ 's, though in principle it can be calculated. However, we are more interested in finding the value of $\lambda_{1}$, which depends solely on the values of $c$ and $\psi$. Instead of solving equation (32) $\lambda_{1}$ can be found by iterating equations (29) and (31) and is equal to their ratio once it has become effectively constant. This was done for a range of $\psi$ and $c$ values to find the magnitude of the effect that worker produced males cause. It is apparent from table 2 that for a given

TABLE 2

The major root governing the speed of approach to equilibrium at two loci for various values of $c$ and $\psi$. This is the rate at which $D$ and I become zero and it is the same for both. An asterisk denotes that the values of $D$ and I oscillate around zero (see text)

\begin{tabular}{cccccccccccc}
$c$ & \multicolumn{110}{c}{$\psi$} & \multicolumn{10}{c}{$\boldsymbol{1 0}$} \\
0.01 & 0.993 & 0.988 & 0.983 & 0.979 & 0.976 & 0.974 & 0.973 & 0.976 & 0.978 & 0.985 & 0.995 \\
0.05 & 0.996 & 0.962 & 0.959 & 0.956 & 0.953 & 0.952 & 0.952 & 0.954 & 0.958 & 0.964 & 0.974 \\
0.10 & 0.933 & 0.929 & 0.927 & 0.925 & 0.924 & 0.924 & 0.925 & 0.927 & 0.931 & 0.938 & 0.947 \\
0.15 & 0.898 & 0.896 & 0.895 & 0.895 & 0.894 & 0.894 & 0.896 & 0.899 & 0.904 & 0.910 & 0.918 \\
0.20 & 0.863 & 0.862 & 0.861 & 0.861 & 0.862 & 0.864 & 0.866 & 0.870 & 0.874 & 0.880 & 0.887 \\
0.25 & 0.828 & 0.827 & 0.827 & 0.828 & 0.829 & 0.832 & 0.835 & 0.838 & 0.843 & 0.848 & 0.854 \\
0.30 & 0.792 & 0.791 & 0.791 & 0.793 & 0.795 & 0.798 & 0.801 & 0.805 & 0.809 & 0.812 & 0.816 \\
0.35 & 0.755 & 0.754 & 0.754 & 0.756 & 0.758 & 0.761 & 0.764 & 0.768 & 0.771 & 0.773 & 0.774 \\
0.40 & 0.718 & 0.716 & 0.715 & 0.716 & 0.719 & 0.721 & 0.724 & 0.727 & 0.728 & 0.728 & 0.724 \\
0.45 & 0.680 & 0.676 & 0.674 & 0.674 & 0.675 & 0.677 & 0.679 & 0.680 & 0.678 & 0.672 & 0.658 \\
0.50 & 0.640 & 0.633 & 0.629 & 0.627 & 0.626 & 0.625 & 0.624 & 0.620 & 0.611 & 0.591 & 0.500 \\
0.55 & 0.600 & 0.588 & 0.578 & 0.571 & 0.564 & 0.556 & 0.545 & 0.520 & $*$ & $*$ & $*$
\end{tabular}

value of $c$ the effect is not very large. For $c<0.45$ intermediate values of $\psi$ accelerate the approach to equilibrium but at high values of $\psi$ the approach is retarded. For $c>0.45$ worker produced males always increase the speed with which equilibrium is attained, but it never equals or exceeds the speed in the corresponding autosomal diplo-diploid case.

Recombination rates in excess of 0.5 are theoretically possible (Owen, 1949) and examples have been found for sex-linked genes in diploids (Wright, 1947). Without seeking to investigate the question in detail it is sufficient to note that for sufficiently large values of $\psi$ values of $c$ in excess of 0.5 have the effect of rendering more complicated the mode by which $D$ and $I$ tend to zero. For example, with $\psi=0.8$ and $c=0.55$, although equilibrium is approached more swiftly than when $c=0.50$, the values of $D$ and $I$ oscillate around zero, changing sign about every 25 generations.

The result for $\psi=0$ (corresponding to the normal sex-linked case in diplo-diploids) is worth deriving here because the method used is different 
from those previously published (Wright, 1933; Bennett, 1963). When $\psi=0$ equation (29) reduces to $I^{\prime \prime}=D^{\prime}+\frac{1}{2}(1-c) I^{\prime}$, equation (31) reduces to $D^{\prime \prime}=\frac{1}{4} D^{\prime}+\frac{1}{4} D+\frac{1}{4}(1-2 c) I^{\prime}$, and the eigenvalue equation $(32)$ becomes $\left[\lambda^{3}-\right.$ $\left.\left(\frac{3}{4}-\frac{1}{2} c\right) \lambda^{2}-\frac{3}{8}(1-c) \lambda+\frac{1}{8}(1-c)\right]=0$, which has roots

$$
\begin{aligned}
& \lambda_{1}=\frac{1}{4}[(1-c)+\sqrt{(1-c)(9-c)}], \\
& \lambda_{2}=\frac{1}{2}(1-c)-\frac{1}{4}[(1-c)+\sqrt{(1-c)(9-c)}], \\
& \lambda_{3}=\frac{1}{2} c,
\end{aligned}
$$

as given by Bennett (1963). The first column of table 2 for $\psi=0$ gives the values of $\lambda_{1}$; for example, when $c=0.5, \lambda_{1}=\frac{1}{4}(0 \cdot 5+\sqrt{4.25})=0.640$.

For a haplo-diploid species the rate of approach to linkage equilibrium is about two-thirds that of a diplo-diploid species with the same recombination rate (Bennett, 1963), and the presence of worker produced males has only a marginal effect.

\section{Discussion}

The main conclusion reached is that worker produced males affect most aspects of social hymenopteran population genetics. The direction of this effect is not, in most cases, intuitively obvious and the equations involving $\psi$

\section{TABLE 3}

Summary of the effects of worker produced males on some aspects of the population genetics of the social Hymenoptera

\section{Aspect}

Approach to Hardy-Weinberg equilibrium

Equilibrium between mutation and selection

Elimination of deleterious alleles

Spread of an advantageous allele

Balanced polymorphism

Approach to linkage equilibrium at two loci

\section{Conditions}

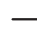

$S_{1}=S_{0}$

$S_{1}>S_{0}$

$S_{1}<S_{0}$

$S_{12}=0$

$S_{12}=0$ and sex-limited

$S_{1}=S_{12}$

$S_{1}<S_{12}$

$S_{1}>S_{12}$

$p \neq P$

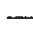

$c<0 \cdot 45$

$c>0.45$

$c=0.55$ and

$\psi \geqq 0.8$
Effect

Rate increased

No change in $p_{e}, P_{e}$

$p_{e}, P_{e}$ increased

$p_{e}, P_{e}$ decreased

$p_{e}, P_{e}$ decreased, genetic load decreased

$p_{e}$ decreased

No change in rate

Rate decreased

Rate increased

Rate of gene frequency change slowed

$P_{e}, p_{e}$ shifted in favour of male genotype with greatest viability. Dependant on relative viabilities polymorphisms extinguished or generated

\section{Moderate values of $\psi$ increases} rate; high values decrease rate Rate increased

Oscillations of $D$ and $I$ around zero 
are often complex and substantially modified from their standard forms. A summary of the population genetic consequences of worker produced males, as analysed here, is given in table 3 . Positive values of $\psi$ usually have an effect whether the population is in equilibrium or not and also in most instances when the gene frequencies in the sexes are equal or unequal.

A particularly interesting consequence of having worker produced males is that in the hymenopteran species in which they occur there may be a marginally higher frequency of polymorphisms than in those species that do not have mixed male parentage. This is because under certain selective regimes quite low values of $\psi$ can generate polymorphisms whereas high values, which are less likely to occur, are required to extinguish them (see table 1). Comparison could be made between social and solitary hymenoptera or between a particular social species and its social parasite which has no worker caste and hence no worker produced males. For instance, in Britain many species of the parasitic genus Psithyrus are highly host specific on the true bumble bees Bombus. Some species in fact only attack one host species; for example $P$. barbutellus on $B$. hortorum, $P$. rupestris on $B$. lapidarius, $P$. vestalis on $B$. terrestris, $P$. bohemicus on $B$. lucorum (Plowright and Owen, 1980). The parasite is subjected to almost the same environmental influences as the host because it is necessarily found within the same geographical area and its brood is attended to by workers of the host species in their nest. Differences between the host and parasite in polymorphism frequencies, etc., therefore could be due to having worker produced males in the host species.

The analysis given in this paper indicates that future experimental and theoretical work concerning the population genetics of the social hymenoptera should take into account the effect of mixed male parentage. Many of the results are applicable to haplo-diploid species of mites where males are initially produced and then mate with females of their mothers' generation.

Acknowledgements.-I thank my father, Dr A. R. G. Owen, for his help and advice with the mathematics, Ms Stella Adams for typing the manuscript, and the Natural Science and Engineering Research Council of Canada for financial support through a Postgraduate Scholarship.

\section{REFERENCES}

BENNETT, J. H. 1957. Selectively balanced polymorphism at a sex-linked locus. Nature, 180, 1363-1364.

BENNETT, J. H. 1963. Random mating and sex-linkage. J. Theor. Biol, 4, 28-36.

CONTEL, E. P. B., AND KERR, W. E. 1976. Origin of males in Melipona subnitida estimated from data of an isozymic polymorphic system. Genetica, 46, 271-277.

CROZIER, R. H. 1976. Why male-haploid and sex-linked genetic systems seem to have unusually sex-limited mutational genetic loads. Evolution, 30, 623-624.

CROZIER, R. H. 1977. Evolutionary genetics of the hymenoptera. Ann. Rev. Entomol., 22, 263-288.

EDWARDS, A. W. F. 1977. Foundations of Mathematical Genetics. Cambridge University Press, Cambridge.

FISHER, R. A. 1930. The Genetical Theory of Natural Selection. Clarendon Press, Oxford. HALDANE, J. B. S. 1935. The rate of spontaneous mutation of a human gene. J. Genetics, 31 , 317-326. 
HALDANE, J. B. S., AND JAYAKAR, S. D, 1964. Equilibria under natural selection at a sex-linked locus. J. Genetics, 59, 29-36.

HAMILTON, w. D. 1964. The genetical theory of social behaviour, I, II. J. Theor. Biol, 7, $1-52$.

HARTL, D. L. 1971. Some aspects of natural selection in arrhenotokous populations. Am. Zool., 11, 309-325.

HARTL, D. L. 1972. A fundamental theorem of natural selection for sex-linkage or arrhenotoky. Am. Nat., 106, 516-524.

LI, C. C. 1967. Genetic equilibrium under selection. Biometrics, 23, 397-484.

MICHENER, C. D. 1974. The Social Behaviour of the Bees: A Comparative Study. Belknap Press of Harvard University Press, Cambridge, Mass.

OSTER, G., ESHEL, I., AND COHEN, D. 1977. Worker-queen conflict and the evolution of social insects. Theor. Pop. Biol., 12, 49-85.

OSTER, G., AND Wilson, E. O. 1978, Caste and Ecology in the Social Insects. Princeton University Press, Princeton, N.J.

OWEN, A. R. G. 1949. The theory of genetical recombination I. Long-chromosome arms. Proc. Roy. Soc., B, 136, 67-94.

OWEN, A. R. G. 1953. A genetical system admitting of two distinct stable equilibria under natural selection. Heredity, 7, 97-102.

PLOWRIGHT, R. C., AND OWEN, R. E. 1980. The evolutionary significance of bumble bee color patterns: A mimetic interpretation. Evolution, 34, in press.

SPRADBERY, J. P. 1973. Wasps: An Account of the Biology and Natural History of Solitary and Social Wasps. University of Washington Press, Seattle.

STILES, E. W. 1979. Evolution of color pattern and pubescence characteristics in male bumble bees: Automimicry vs thermoregulation. Evolution, 33, 941-957.

TRIVERS, R. L., AND HARE, H. 1976. Haplodiploidy and the evolution of the social insects. Science, 191, 249-263.

TURNER, J. R. G. 1968. Natural selection for and against a polymorphism which interacts with sex. Evolution, 22, 481-495.

WRIGHT, M. E. 1947. Two sex-linkages in the house mouse with unusual recombination values. Heredity, 1, 349-354.

WRIGHT, s. 1933. Inbreeding and recombination. Proc. Nat. Acad. Sci., 19, 420-433. 